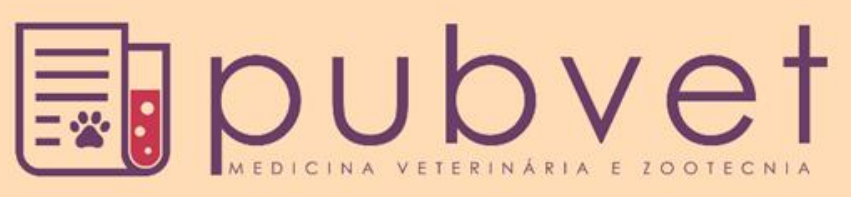

ISSN $1982-1263$

https://doi.org/10.31533/pubvet.v13n6a360.1-5

\title{
Hemangioma conjuntival em cão: Relato de caso
}

\author{
Tatiane Amancio $^{1 *}$, Lívia Gonçalves da Silva Valente $^{2}$, Jonis Baesso Ghizzo ${ }^{1}$, Tainá Souza \\ Gonçalves $^{1 \oplus}$, Laura Vieira Tonon ${ }^{2} \theta$, Luciane Orbem Veronezi ${ }^{3} \theta$, Guilherme Valente de Souza ${ }^{4}$ \\ ${ }^{I}$ Acadêmica de Medicina Veterinária, Centro Universitário Barriga Verde - UNIBAVE. Orleans - SC Brasil. \\ ${ }^{2}$ Médica Veterinária, Pós-graduanda, Universidade do Estado de Santa Catarina - UDESC. Lages - SC, Brasil. \\ ${ }^{3}$ Médica Veterinária Dra Docente, Centro Universitário Barriga Verde - UNIBAVE. Orleans - SC, Brasil. \\ ${ }^{4}$ Médico Veterinário, Doutor, Centro Universitário Barriga Verde - UNIBAVE. Orleans - SC, Brasil. \\ *Autor para correspondência, E-mail: tatiamancio@outlook.com
}

Resumo. As neoplasias oculares podem comprometer a visão e consequentemente a qualidade de vida dos animais, são afecções comuns na clínica de pequenos animais que exigem tratamento e diagnósticos rápidos a fim de, se conseguir uma resposta terapêutica eficiente. O Hemangioma é uma neoplasia de origem do endotélio vascular, benigna, que não apresenta invasão periférica, e pode estar associado à alta incidência de radiação solar. O presente trabalho relata o caso clínico de um canino, da raça Pit Bull, macho, atendido no Hospital Veterinário do Unibave (HVU), com nódulo conjuntival. O tratamento realizado foi à dissecção cirúrgica, com amplas margens, com objetivo de eliminar toda a massa tumoral e garantir uma margem cirúrgica livre de células tumorais. A amostra dissecada foi encaminhada para avaliação histológica e teve como resultado o diagnóstico de hemangioma. $\mathrm{O}$ tratamento cirúrgico foi bem sucedido e o paciente obteve melhora clínica, sem complicações no trans e pós-operatório.

Palavras-chave: Cirurgia, conjuntiva, oftalmologia

\section{Conjunctival hemangioma in dogs: Case report}

Abstract. Ocular neoplasias can compromise eyesight and consequently the quality of life of the animals, are common conditions in the small animal clinic that require treatment and rapid diagnosis in order to achieve an efficient therapeutic response. Hemangioma is a benign vascular endothelial neoplasm with no peripheral invasion and may be associated with a high incidence of solar radiation. This paper reports the clinical case of a male Pit Bull dog, treated at the Unibave Veterinary Hospital (HVU), with a conjunctival nodule. The treatment was surgical dissection, with wide margins, with the aim of eliminating the entire tumor mass and guaranteeing a surgical margin free of tumor cells. The dissected sample was referred for histological evaluation and resulted in the diagnosis of hemangioma. The surgical treatment was successful, and the patient obtained clinical improvement, without complications in the trans and postoperative period.

Key words: Surgery, conjunctiva, ophthalmology

\section{Hemangioma conjuntival en perros: Reporte de caso}

Resumen. Las neoplasias oculares pueden comprometer la visión y consecuentemente la calidad de vida de los animales, son afecciones comunes en la clínica de pequeños animales que requieren tratamiento y diagnóstico rápido a fin de lograr una respuesta terapéutica eficiente. El Hemangioma es una neoplasia de origen del endotelio vascular, benigna, que no presenta invasión periférica, y puede estar asociada a la alta incidencia de radiación solar. El presente trabajo relata el caso clínico de un canino, de la raza Pit Bull, macho, 
atendido en el Hospital Veterinario del Unibave (HVU), con nódulo conjuntival. El tratamiento realizado fue a la disección quirúrgica, con amplios márgenes, con el objetivo de eliminar toda la masa tumoral y garantizar un margen quirúrgico libre de células tumorales. La muestra disecada fue encaminada para evaluación histológica y tuvo como resultado el diagnóstico de hemangioma. El tratamiento quirúrgico fue exitoso y el paciente obtuvo una mejora clínica, sin complicaciones en el trans y postoperatorio.

Palabras clave: Cirugía, conjuntiva, oftalmología

\section{Introdução}

O neoplasma se caracteriza por um novo crescimento composto de células, originada de tecidos normais, mas que sofrem algumas alterações que permitem que elas se tornem relativamente não responsivas ao crescimento normal, ultrapassando os limites anatômicos normais (Zachary et al., 2012). Segundo Zachary et al. (2012), os tumores benignos raramente causam a morte do hospedeiro, e geralmente são curáveis, pois não invadem tecidos vizinhos e nem se espalham para outras localizações. Já os tumores malignos, podem causar metástases, ou seja, pode se espalhar pelo corpo e até causar a morte do hospedeiro (Coelho, 2016).

Segundo Olbertz (2012), a visão é um dos sentidos envolvidos na sobrevivência, qualidade de vida e na percepção dos seres vivos em relação às mudanças que ocorrem no meio que os cerca. Quando ocorre alguma alteração patológica, como tumores em locais como a pálpebra, mesmo sendo benignos e pequenos, podem ocorrer o mau fechamento das mesmas, ocasionando irritação ocular pelo contato direto do tecido neoplásico com a superfície corneana (Olbertz, 2012).

As neoplasias oculares podem acometer todas as espécies domésticas, entretanto se tem predominância em cães e gatos. Um dos anexos oculares é a conjuntiva, sendo uma membrana mucosa, delgada, transparente, ricamente vascularizada, externa entre todas as membranas mucosas, ou seja, recobre a face interna das pálpebras (Santos \& Alessi, 2016). Fatores genéticos, da biologia individual e ambiental podem influenciar a oncogênese, como lesões sofridas pelas pálpebras e pela conjuntiva decorrente da radiação solar (Santos \& Alessi, 2016). De acordo com um estudo realizado por Gomes (2015) após análise estatística concluiu-se que não existem diferenças significativas em ambas às espécies entre idade e neoplasias em cada estrutura ocular, e as neoplasias com maior incidência na conjuntiva canina foram o hemangioma e o papiloma escamoso.

O hemangioma e o hemangiossarcoma são neoplasias de origem do endotélio vascular, e podem surgir no interior da lâmina própria conjuntival da borda lateral da terceira pálpebra, e na conjuntiva bulbar lateral. $\mathrm{O}$ hemangioma se caracteriza por ser um tumor benigno, bem circunscrito e consistem em um endotélio delicado, já o hemangiossarcoma descrito como maligno, é formado por um endotélio hipercromático com moderada anisocariose e invasão periférica (Zachary et al., 2012). Tem-se uma sequência histológica de hemangioma até hemangiossarcoma, mas um mesmo tumor pode apresentar a sequência de maligno para benigno. Portanto, a classificação de benigno e maligno, se dá pelo grau de invasão periférica (Zachary et al., 2012). Deve ser considerado em todos os casos suspeitos, um diagnostico completo através de técnicas de diagnóstico por imagem, citologia e biópsia. A excisão de todo o tecido neoplásico é frequentemente curativa (Conceição et al., 2010). Segundo Santos \& Alessi (2016) alguns estudos sobre hemangiomas conjuntivais primários em cães, revelou que há tendência da neoplasia recidivar após a cirurgia, mas metástases são extremamente raras, e a incidência do animal apresentar hemangioma bilateral, ocorre cerca de $30 \%$, e animais que vivem em regiões com alta incidência de radiação solar e elevada altitude, tem maior tendência a desenvolvê-lo.

Nesse presente trabalho, iremos relatar um caso de hemangioma canino, que foi atendido no Hospital Veterinário do UNIBAVE.

\section{Procedimentos metodológicos}

No dia três de novembro de 2017 foi atendido no Hospital Veterinário do Unibave - HVU, na cidade de Orleans, um cão, macho, da raça American Pit Bull Terrier, de 5 anos, apresentando um nódulo ulcerado na conjuntiva, associado também com hiperplasia da terceira pálpebra. 
Durante a anamnese, o tutor relatou que o paciente não tinha suas vacinas em dia, à última desvermifugação havia sido realizada há um ano com ivermectina (dose não informada), nunca havia evidenciado patologias anteriores, apresentava normodipsia, normúria, normoquesia, normourequesia e que havia visualizado um nódulo no olho do canino há aproximadamente 15 dias com crescimento progressivo e sangramento há dois dias.

Durante o exame clínico do paciente, foi verificada a presença da massa neoplásica ulcerada na conjuntiva abaixo da pálpebra superior direita (Figura 1). Os demais exames clínicos apresentaram parâmetros dentro da normalidade para a espécie referida. Foram coletadas amostras sanguíneas para hemograma e bioquímicos, e aspirado do nódulo por punção aspirativa por agulha fina (PAAF), para confecção de lâminas para citologia. Tanto o hemograma como os bioquímicos, não apresentaram valores diferentes dos de referência para caninos, já o laudo citológico sugeriu processo inflamatório predominantemente neutrofílico.

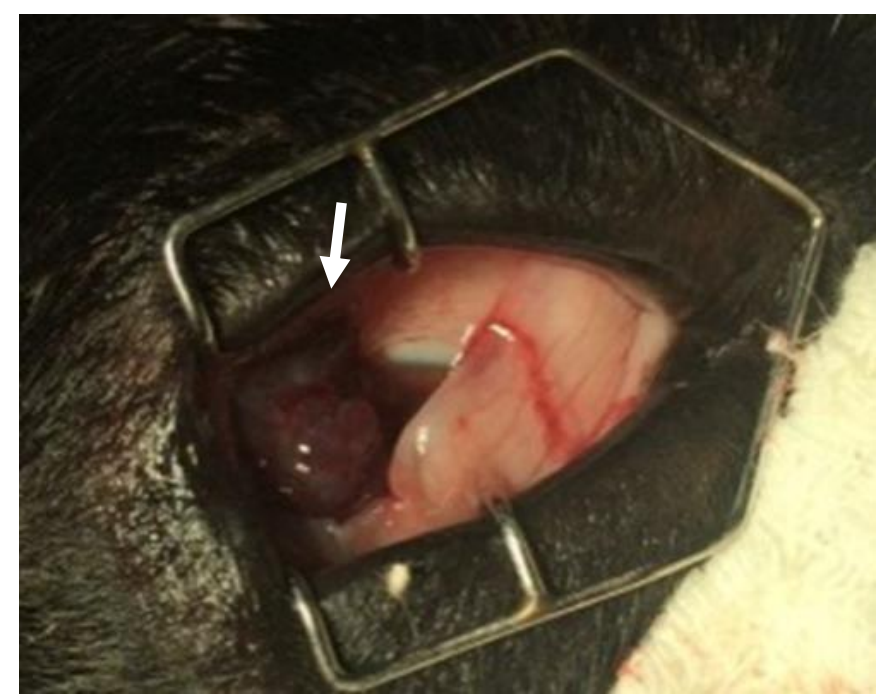

Figura 1. Imagem do olho direito, apresentando nódulo ulcerado (Seta branca). Fonte: Hospital Veterinário UNIBAVE, 2017.

Diante ao caso, houve comum acordo entre o proprietário e o cirurgião de efetuar o ato cirúrgico para dissecação do nódulo, para proporcionar conforto para o paciente e realizar o histopatológico com o objetivo de se obter um diagnóstico conclusivo.

Foi realizado o procedimento cirúrgico, realizando a dissecação ao redor do tumor, com o objetivo de conseguir margens cirúrgicas com segurança, livres de células tumorais. Sendo utilizado para a hemostasia dos vasos sanguíneos, o cauterizador elétrico. Após o procedimento, da total ressecção do tumor (figura2), o nódulo foi fixado em formol a 10\%, identificado e encaminhado para o laboratório de patologia animal do UNIBAVE - HVU, onde foi realizado o exame histopatológico.

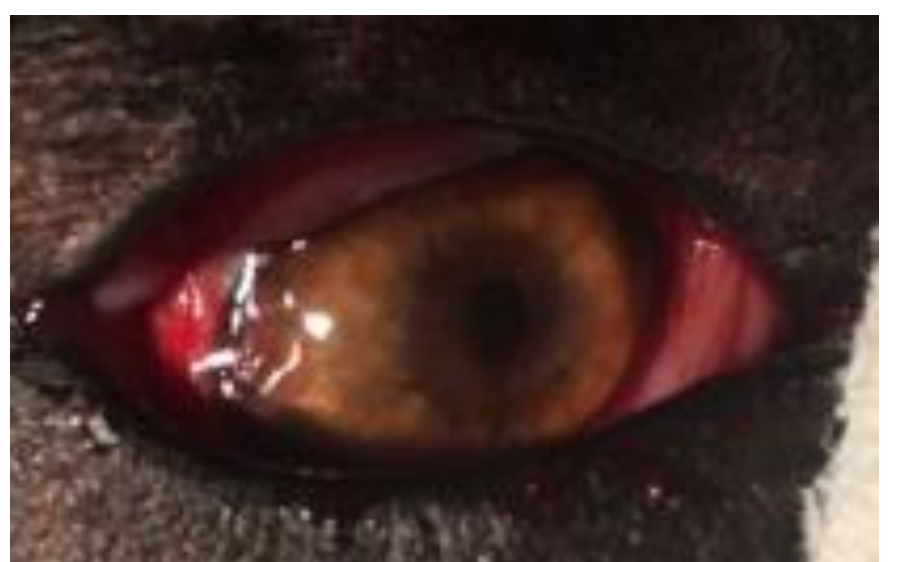

Figura 2. Imagem do olho direito, após a retirada do nódulo no pós-cirúrgico imediato. Fonte: Hospital Veterinário UNIBAVE, 2017. 
A massa encaminhada para o histopatológico media $1,2 \mathrm{~cm}$ de comprimento, por $0,5 \mathrm{~cm}$ de largura $\mathrm{e}$ $0,4 \mathrm{~cm}$ de altura (figura 3). Ao corte continha consistência macia e coloração avermelhada. No laudo histopatológico, observou-se a proliferação de células endoteliais moderadamente distendidas, que formam vasos sanguíneos e capilares irregulares com um lúmen variando de pequeno a grande ao longo de toda a extensão ocupada pela massa. Obtendo-se lesões histológicas que caracterizam um hemangioma cavernoso (Figura 4).

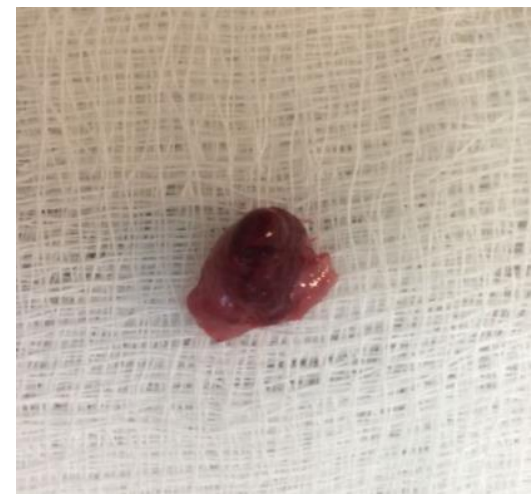

Figura 3. Massa retirada cirurgicamente e posteriormente encaminhada para o histopatológico. Fonte: Hospital Veterinário UNIBAVE, 2017.
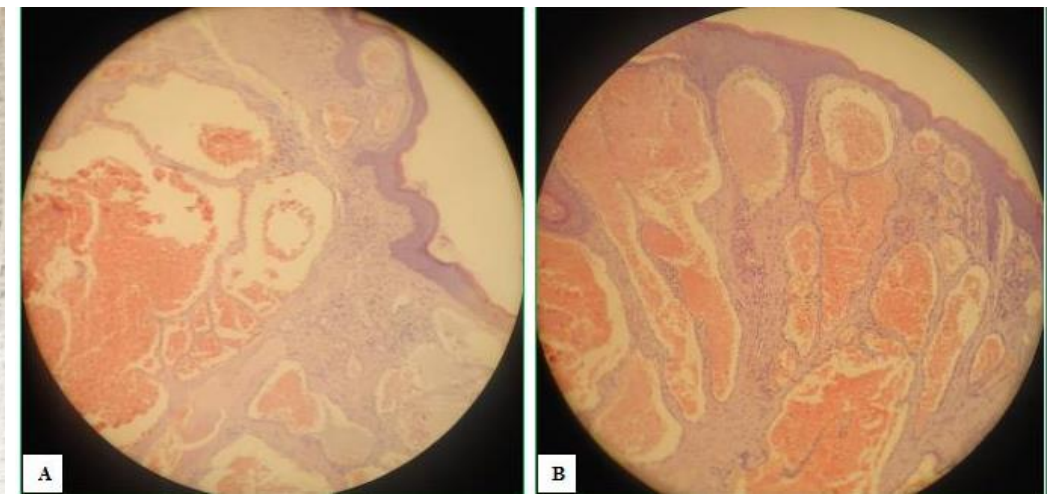

Figura 4. Imagem do exame histopatológico. Hemangioma Cavernoso (A) e proliferação de células endoteliais formando vasos sanguíneos e canais vasculares irregulares, preenchidos com eritrócitos, com lúmen de pequeno a grande (B). Coloração: H\&E. Obj. 10x. Fonte: Hospital Veterinário UNIBAVE, 2017.

O animal recebeu alta no dia seguinte do procedimento e o tratamento pós-operatório foi orientado ao proprietário. A prescrição foi composta de anti-inflamatório oral a base de meloxican $0,1 \mathrm{mg} / \mathrm{Kg}$, uma vez ao dia (SID), durante 3 dias e tratamento tópico com colírio (Maxitrol ${ }^{\circledR}$ ) de dexametasona 0,001 $\mathrm{g}+$ sulfato de neomicina $0,005 \mathrm{~g}+$ sulfato de polimixina B $6000 \mathrm{UI}+$ hipromelose $0,005 \mathrm{~g} \mathrm{em} 1 \mathrm{~mL}$ de veículo, quatro vezes ao dia (QUID), durante 7 dias. Também foi recomendada a utilização do colar elisabetano, durante 10 dias.

O paciente retornou 10 dias após o procedimento e o animal apresentava uma boa recuperação pós-cirúrgica, tendo ocorrido uma boa cicatrização da conjuntiva. Demonstrando um procedimento cirúrgico de sucesso.

\section{Resultados e discussão}

Segundo estudo de casos realizado por Camargo et al. (2008), Paranhos (2014) e Hesse et al. (2015) as neoplasias benignas em caninos representaram o maior número de diagnósticos, ou seja, destacando maior frequência, encaixando-se assim, o presente caso de hemangioma, como mais comum que o hemangiossarcoma.

Durante o estudo realizado por Soares et al. (2017), com 192 cães, o hemangioma foi diagnosticado em 64 cães, sendo 37 fêmeas e 27 machos. Foi também relatado que as raças labradror e golden retriever são as mais acometidas por hemangioma, já em relação ao hemangiossarcoma a raça com maior predisposição é a American Pit Bull Terrier estando em desacordo com o presente trabalho em que se tratava de um cão da raça American Pit Bull Terrier, com hemangioma e que não está na relação de raças, mais acometidas por este tipo de neoplasia.

Como citado por Santos \& Alessi (2016), os animais que vivem sobre alta incidência de radiação solar, podem ser os mais acometidos, onde destacamos uma das possíveis causas para o paciente do caso, pois se tratava de um canino domiciliado e com acesso constante ao sol.

Para neoplasias do endotélio vascular, a recomendação de tratamento para estes tumores é a excisão cirúrgica precoce e ampla para se obter margens. Tal cirurgia pode ser curativa, embora a recorrência seja possível (Pirie et al., 2006). Conforme assim, foi esse o objetivo na realização do tratamento do paciente, sendo o procedimento cirúrgico ocorrido, e com sucesso. 


\section{Considerações finais}

As neoplasias conjuntival, mesmo quando ocorridas da forma benigna, é importante a retirada da massa, priorizando o bem-estar do animal e qualidade de vida. Destaca-se a retirada cirúrgica como melhor protocolo terapêutico, para obter-se melhor prognóstico.

\section{Referências bibliográficas}

Camargo, L. P., Conceição, L. G. \& Santos Costa, P. R. (2008). Neoplasias melanocíticas cutâneas em cães: estudo retrospectivo de 68 casos (1996-2004). Brazilian Journal of Veterinary Research and Animal Science, 45(2):138-152.

Coelho, H. E. (2016). Patologia veterinária. Uberaba, Minas Gerais: Coelho.

Conceição, L. F., Ribeiro, A. P., Piso, D. Y. T. \& Laus, J. L. (2010). Considerations about ocular neoplasia of dogs and cats. Ciência Rural, 40(10):2235-2242.

Gomes, D. S. P. (2015). Neoplasias oculares do cão e gato: estudo retrospectivo de 5 anos. Master of Science, Universidade Lusófona de Humanidades e Tecnologias, Lisboa, Portugal.

Hesse, K. L., Fredo, G., Guimarães, L. L. B., Reis, M. d. O., Pigatto, J. A. T., Pavarini, S. P. \& Sonne, L. (2015). Neoplasmas oculares e de anexos em cães e gatos no Rio Grande do Sul: 265 casos (20092014). Pesquisa Veterinária Brasileira, 35(1):49-54.

Olbertz, L. (2012). Levantamento clínico-epidemiológico com análise morfológica das principais neoplasias oculares em cães. Universidade Federal do Paraná, Curitiba, Paraná, Brasil.

Paranhos, C. A. (2014). Neoplasias cutâneas caninas: um estudo descritivo de 4 anos. Master of Science, Universidade de Trás-os-Montes e Alto Douro, Vila Real.

Pirie, C. G., Knollinger, A. M., Thomas, C. B. \& Dubielzig, R. R. (2006). Canine conjunctival hemangioma and hemangiosarcoma: a retrospective evaluation of 108 cases (1989-2004). Veterinary Ophthalmology, 9(4):215-226.

Santos, R. L. \& Alessi, A. C. (2016). Patologia veterinária. Rio de Janeiro: Brasil: Roca.

Soares, N. P., Medeiros, A. A., Szabó, M. P. J., Guimarães, E. C., Fernandes, L. G. \& Santos, T. R. (2017). Hemangiomas e hemangiossarcomas em cães: estudo retrospectivo de 192 casos (20022014). Ciência Animal Brasileira, 18(e-30889):1-10.

Zachary, J. F., McGavin, D. \& McGavin, M. D. (2012). Bases da patologia em veterinária. Rio de Janeiro: Elsevier Brasil.

Recebido: 6 de maio, 2019.

Aprovado: 11 de junho, 2019.

Publicado: 3 de julho, 2019.

Licenciamento: Este artigo é publicado na modalidade Acesso Aberto sob a licença Creative Commons Atribuição 4.0 (CCBY 4.0), a qual permite uso irrestrito, distribuição, reprodução em qualquer meio, desde que o autor e a fonte sejam devidamente creditados. 